\title{
The visual accommodation response during concurrent mental activity
}

\author{
FREDERICK V. MALMSTROM \\ Institute of Safety and Systems Management, University of Southern California \\ Los Angeles, California 90007 \\ ROBERT J. RANDLE \\ NASA/Ames Research Center, Moffett Field, California 94035 \\ JOHN S. BENDIX \\ Amherst College, Amherst, Massachusetts 01002 \\ and \\ ROBERT J. WEBER \\ Oklahoma State University, Stillwater, Oklahoma 74074
}

\begin{abstract}
Concurrent mental activity seems to be a significant, nonvisual factor affecting the human accommodation response. Two experiments were conducted to determine the direction and magnitude of this accommodation response. Experiment 1 employed a concurrent, written backwards counting task. Experiment 2 employed a concurrent, mental imagery task of "thinking near" and "thinking far." In both experiments, the concurrent secondary task effected a cumulative accommodative shift toward the visual far point of from .25 to .75 diopter away from a near (3.0 diopter) target. This accommodative shift was observed only in the presence of a stimulus field and not in open-loop (analogous to empty-field) conditions. In addition, a long-term instability in the open-loop method of obtaining the dark focus was observed. Similarities between this accommodative shift and the pupillary response are noted. The accommodation response is discussed in relationship to both an attention-sharing and an involuntary autonomic response model.
\end{abstract}

For the past 40 years, there has been some attempt to investigate the nonvisual factors that can affect the visual accommodation response. Some of the suggested variables affecting accommodation have been autonomic arousal states, stress, mood shifts, and visual imagery (Malmstrom \& Randle, 1976; Miller, 1978; Morgan, Olmsted, \& Watrous, 1940;

This research was conducted while the first and third authors were guest workers at the NASA/Ames Research Center. All work was performed in fulfillment of the U.S. Air Force Systems Command's Mobilization Augmentee Program. Portions of Experiment 1 are contained in the first author's doctoral thesis, Oklahoma State University. Support was provided by funding from the Man-Vehicle Systems Research Division, NASA/Ames Research Center, the USAF/Rome Air Development Center, and from National Science Foundation Grant 74-20208 to the last author. However, no endorsement of any agency should be implied. The authors express their appreciation to Donna L. Miller, Informatics, Inc., for her assistance in the operation of the Cornsweet and Crane optometer, to James Price for help and suggestions in the data analysis, and to Susan Gates for administrative assistance. Requests for reprints should be sent to F. V. Malmstrom, Human Factors Department, Institute of Safety and Systems Management, University of Southern California, Los Angeles, California 90007 . John Bendix's present address is 3 Orchard Land, Berkeley, California 94704.
Olmsted \& Morgan, 1939; Westheimer, 1957).

Objective measures of accommodation are not easy to obtain. Buried beneath the cornea, aqueous humor, and iris, the lens does not exhibit many changes that can be observed either objectively or unobtrusively. It has traditionally been necessary to query the subject directly as to whether an image is in focus, for example, with a Badal optometer or a Snellen chart. Thus, it has been nearly impossible to separate pure accommodation changes from the more subjective elements of perception.

On the other hand, the well-documented pupillary response (Hess, 1975) has been relatively easy to observe and has been considered by some researchers to be a reliable indicator of stress, emotion, and autonomic arousal (Ahern \& Beatty, 1979; Hess \& Polt, 1960; Kahneman \& Beatty, 1966).

A recent study by Randle, Roscoe, and Petitt (Note 1) examined 20 commercial pilots who were required to make several task-related decisions during a simulated night-landing task while viewing the display through various magnifications. The results revealed an unexpected effect; the increasing importance of the decision appeared to influence 
the accommodative state of the pilot. Each cumulative flight decision revealed a small (about .1 diopter; $\mathrm{D}=\mathrm{m}^{-1}$ ), but persistent, accommodative shift, always toward the visual far point. There are at least two possible interpretations of these results. First, it is possible that some amount of increasingly important concurrent mental task could have brought about an increasing distraction from the primary visual tracking task. Such an interpretation would be compatible with the model proposed by Kahneman (1973) which allows for only a limited amount of processing capacity. Second, it is possible that the accommodative shift observed would be reflective of a task-evoked autonomic response, similar to the pupillary response noted by Hess and Polt (1964).

In 1954, Schober proposed that near accommodation reflected the properties of a parasympathetic response; likewise, far accommodation reflected the properties of a sympathetic response. Indeed, a parallel (but simplified) description of the pupillary response says that, while pupillary dilation is a sympathetic response, pupillary constriction is a parasympathetic response (Davson, 1976). Since both the accommodation and pupillary (as well as the convergence) responses have long been known to operate quasi-independently, as in the well-documented "near reflex" (Davson, 1976), it might be well to question whether the accommodation response might be equally as responsive to nonvisual stimuli as the pupillary response.

If accommodation shifts are to be observed as a function of concurrent mental tasks, then it seems worthwhile to consider the dark focus, or the intermediate resting position of accommodation, first proposed by Cogan (1937) and later discussed and expanded upon by Hennessy (1975), Leibowitz and Owens (1975), Malmstrom and Randle (1976), Owens (1979), and Schober (1954). Under the constraints of this hypothesis, it is proposed that the dark focus is a behavioral null between the sympathetic and parasympathetic systems. Although the dark focus varies widely from subject to subject (Leibowitz \& Owens, 1975) and even from day to day (Miller, 1978), the value obtained for most subjects ranges between 1.0 and $2.0 \mathrm{D}$. It would be reasonable to expect that any nonvisual changes in accommodation should vary about the dark focus, according to any secondary task demands. However, because Randle, Roscoe, and Petitt (Note 1) and Malmstrom, Randle, and Weber (Note 2) had noted that there also appeared to be accommodation shifts in the presence of a focusable target, both the darkfocus and the fixed-focus target responses needed to be examined during the course of some concurrent mental activity.

\section{EXPERIMENT 1: CONCURRENT BACKWARDS COUNTING TASK}

\section{Method}

Subjects. The subjects were five naive males, aged 21 to 23 years and with at least 20/20 vision (near and far), as determined by a Bausch and Lomb orthorater. The age requirement was to insure a wide range of accommodation. All subjects were paid hourly for participating in five consecutive daily experimental sessions lasting about $1 \mathrm{~h}$ each. A further determination of the subjects' near and far points was made using the attached focus stimulator as a subjective Badal optometer (see Apparatus section). Measurements were monocular, left eye only, averaged over five trials taken at the beginning of the first day. In addition, for the subjects examined in Experiment 1, a determination of their dark focus (open-loop accommodation) was made for the second minute of a 2-min open-loop viewing condition. The dark-focus value is an average of $101-\mathrm{min}$ data points, taken once at the beginning and once at the end of each of the 5 days. The near and far points along with the openloop dark-focus values are shown for each subject in Table 1.

Apparatus. All measurements were taken with the Cornsweet and Crane (1970) objective optometer at the NASA/Ames Research Center. Two pieces of apparatus were used. The first, the optometer, is an infrared, continuously nulling (servocontrolled) device based on the familiar Scheiner principle (Davson, 1976). A retinally reflected image of a rectangular aperture is optically made to fall on a split-field photosensor. A difference in focus between the optometer and the subject's eye results in a lateral displacement (motion) of the rectangle on the photosensor from which an error signal of appropriate sign and magnitude is developed. This error signal drives a small high-speed servo on the optometer to a new position that nulls the error signal, which results in the difference in focus. The second piece of equipment, the focus stimulator, is a variation of the basic Badal arrangement. (For a description of the Badal optometer, see Ogle, 1961.) Movement of the target towards the lens requires positive accommodation by the subject; movement away from the lens requires negative accommodation. The focus stimulator used here is similar, except that the target remains stationary and its image is made the object for the viewing eye. Movement of the prism then changes the position of the image; the prism is mounted on a servomotor exactly like that used in the optometer. The focus stimulator is described in detail elsewhere (Crane \& Cornsweet, 1970). The subject's head was held in place by a hot-wax molded bite board affixed to the optometer chassis. Hence, any intelligible conversation by the subject was

Table 1

Subjects' Near and Far Points and Open Loop Dark Focus (in Diopters)

\begin{tabular}{lrrrr}
\hline & \multirow{2}{r}{$\begin{array}{c}\text { Far } \\
\text { Subject }\end{array}$} & $\begin{array}{l}\text { Near } \\
\text { Point }\end{array}$ & Point & \multicolumn{2}{c}{ Open Loop Dark Focus* } \\
\cline { 5 - 6 } M.M. & -.5 & 4.4 & -.50 & SD \\
S.Q. & -.8 & 3.2 & -.17 & .53 \\
D.S. & -.4 & 4.0 & 2.83 & .67 \\
R.L. & -1.0 & 3.6 & 1.93 & 1.12 \\
R.M. & .0 & 3.0 & 1.27 & 1.12 \\
J.N. & .5 & 3.5 & & \\
\hline
\end{tabular}

Note-Subject R.M. participated in Experiment 1 only. Subject J.N. participated in Experiment 2 only. *Overall mean = $1.07(S E=1.40)$ 
impossible unless he first pulled away from the optometer. All measurements were taken from the left eye; the right eye was occluded.

Stimuli. All subjects were presented with a black " $x$ " target subtending a constant visual angle of $2.9 \mathrm{deg}$. The target was presented under three separate constant-focus conditions, $0.0 \mathrm{D}$ (far), 3.0 D (near), and open loop (indeterminate distance). The near and far targets were presented relative to the subject's previously measured far point using the attached focus stimulator (Crane \& Cornsweet, 1970) as a subjective Badal optometer. During the open loop conditions, a selsyn-operated .3-mm aperture was dropped into place at a plane of the entrance pupil of the subject's eye. Concurrently, the $\times$ target background brightness was increased to maintain constant target illumination. The eye's entrance pupil thus became $.3 \mathrm{~mm}$, a condition which increased the depth of field to an extent that the target was always in clear focus. Retinal blur and blur changes were now absent, and a condition now existed which was analogous to empty-field conditions, inasmuch as accommodation was not required. For all conditions, the target background brightness remained constant at about $140 \mathrm{~cd} / \mathrm{m}^{2}$, well above the luminance level required for accommodation.

Instructions and Procedure. Each subject was informed that this was an experiment designed to measure his ability to maintain focus on a steady target and perform a secondary backwards counting task. However, he should treat the focusing and counting tasks with equal importance. At no time during the experiment was the subject informed of the optical distance of the $\times$ target, nor was he given any feedback on whether he had done well or poorly on either the primary (tracking) or the secondary (counting) task until after completion of both experiments.

The secondary task was a paced, backwards counting, written task, recorded by having the subject write his responses on a tablet placed by his writing arm. During the first $30 \mathrm{sec}$, the subject maintained steady focus on the $\times$ target and no counting response was required. At the end of the $30-\mathrm{sec}$ baseline period, the subject was instructed, "Write backwards by ones, start at ... (random two-digit number)." At this point, a randomly selected two-digit number was announced to the subject, and the subject began a paced counting task, writing one count (but two digits) every $2 \mathrm{sec}$. The subject paced his counting by using the audible 1 -sec clicks of the time base on the Brush recorder. For example, if the experimenter said, "Seventy-one," at that instant the subject wrote " 7 " during the first second, "1" during the second second, " 7 " during the third second, " 0 " during the fourth second, " 6 " during the fifth second, " 9 " during the sixth second, and so on. At the end of the 30-sec period, the subject was instructed to stop counting.

During the open-loop tracking conditions, the subject was first given $1 \mathrm{~min}$ of viewing the target in open-loop conditions on the assumption that preaccommodation to an object of indeterminate focus would wash out any residual "biological hysteresis" (Randle, 1975). At the end of the first minute of preaccommodation to the open-loop $\times$, the actual trial was begun.

A previous study (Malmstrom \& Randle, 1976) had suggested that long-term responses to open-loop conditions were rather slow and subject to artifacts. Therefore, it was decided to use an average rather than an instantaneous response measure as in the previous study. Therefore, each 5 -sec time block is actually an average of five instantaneous diopter readings, taken at a rate of $1 / \mathrm{sec}$ from $2.5 \mathrm{sec}$ before each time block to $2.5 \mathrm{sec}$ after each time block. The averaging procedure was intended to smooth out artifacts such as eyeblinks, inadvertent saccades, and the natural $2-3-\mathrm{Hz}$ accommodative oscillations.

Design. Presentations of open-loop, 3.0-D, and 0.0-D targets were in random order within each session. The experiment was entirely a within-subjects design utilizing five subjects over 5 days. The variables were the 2 tasks (track target only, track and count) by 3 target distance conditions $(0.0 \mathrm{D}, 3.0 \mathrm{D}$, open loop) by 7 time blocks $(0-30 \mathrm{sec})$ by 5 days. The terminology "track the target" was used with the subjects instead of the perhaps more accurate terminology "maintain focus" because the phrase was more intelligible to the subject. In any event, the target itself did not move, and the subjects understood the directions to mean "maintain focus." Because the "track-targetonly" task was used to establish baseline accommodative conditions, the order of the two tasks was always fixed. However, there was an approximately 5-sec gap between the two tasks while the subject received the instructions, "Count backwards by ones. Start at ...."

\section{Results and Discussion}

Figure 1 contains the mean accommodative response as a function of the three target distances and the two tasks (track only, count and track) taken over the entire 30 -sec period.

There are, of course, distinct differences between the initial target distance conditions $(0.0 \mathrm{D}, 3.0 \mathrm{D}$, open loop). However, this is both an expected and a trivial effect, indicating that when a target is set at a particular optical distance, the eye tends to focus on it. Therefore, this result will not be discussed further.

Consider next the apparent shift of the accommodation response away from the 3.0-D (near) target during the concurrent task. This effect is not only consistently away from the near target, but also appears to intensify during the course of the secondary counting task.

An overall analysis of variance for the experiment shows a significant main effect for the target distance $[F(2,8)=16.144, p<.002]$. There was a single two-way interaction of Tasks by Target Distance $[F(2,8)=8.609, p<.02]$, and there was a single three-way interaction of Time Blocks by Tasks by Target Distance $[\mathrm{F}(12,48)=2.392, \mathrm{p}<.02]$.

Because the variances from performance on the

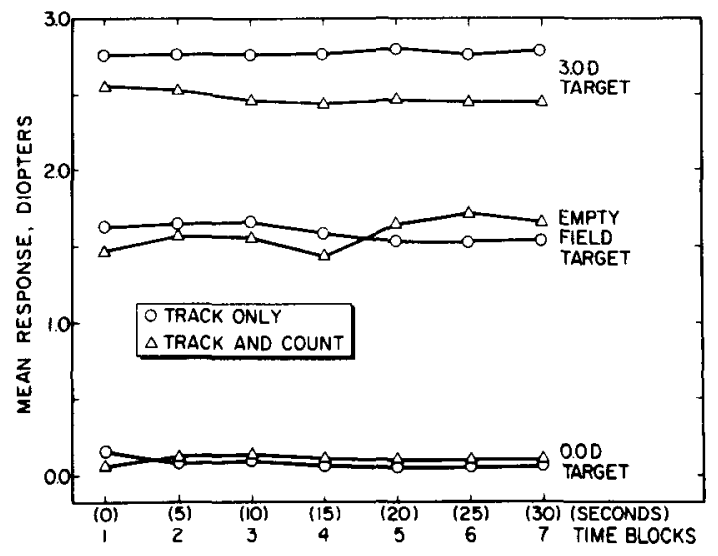

Figure 1. Experiment 1. Concurrent counting task. Mean accommodation response plotted as a function of task and target distances; $\mathbf{N}=\mathbf{5}$. 
open-loop condition were quite large, it was decided to perform a separate analysis of variance using only the two fixed-focus targets (0.0 and 3.0 D). Analysis of variance for this portion of the experiment showed a similar main effect for target distance conditions $[F(1,4)=264.375, p<.001]$. There was also a similar two-way interaction effect for the two tasks $[F(1,4)=10.017, p<.04]$. And, in addition, there were three-way interactions of Time Blocks by Tasks by Target Distances $[F(6,24)=3.883, p<.01]$ and Time Blocks by Target Distances by Days $[F(24,96)$ $=1.840, \mathrm{p}<.025]$. There were no other main interaction effects.

When the open-loop condition is dropped from the analysis of variance, the three-way interaction of Time Blocks by Tasks by Target Distances is strengthened. Considering the loss of degrees of freedom $(12,48$ vs. 6,24$)$, this increased effect is unexpected and gives rise to several implications: The long-term dark focus, at least as measured by the open-loop technique, does not appear to be ideally suited for use in measuring changes in accommodation induced by secondary tasks. Open-loop dark-focus changes appear to be highly unstable and subject to unaccounted-for effects, and so the apparent open-loop interaction effect (see Figure 1) should probably be disregarded. It would, of course, be worthwhile to compare each individual's overall dark focus with his open-loop accommodation shifts during the concurrent task. However, the overall dark focus, as shown by the standard deviations in Table 1, is, itself, highly unstable and subject to trial-to-trial and day-to-day variations. One subject (R.L.), for instance, exhibited as much as a 3.1-D (from Days 3 to 5) dark-focus shift.

Two reasons for this apparent open-loop darkfocus instability have been suggested. First, it may be that dark focus measured in total darkness is different from dark focus measured from a pinhole aperture. This would seem to be unlikely in view of the high degree of correlation between the night and empty-field values observed by Leibowitz and Owens (1975). Another reason, suggested by Roscoe and Benel (Note 3 ) is that long-term (1 min or longer) empty-field and open-loop accommodation responses may be radically different from short-term responses. In this 1978 study, Roscoe and Benel noted a great deal of accommodative wander when the eye was subjected to open-loop conditions for periods of from 1 to $4 \mathrm{~min}$. Miller (1978), on the other hand, found a high degree of intrasubject dark-focus stability. However, the methods of obtaining the dark focus and the data-recording procedures (instantaneous vs. continuous) were substantially different in both experiments.

Disregarding the open-loop accommodation data, there is a two-way interaction effect for Target
Distance by Tasks which warrants comment. This effect indicates that there is a task-induced shift consistently away from the subjects' focus on the near target towards the visual far point. Also, there is an apparent gap between the end of the track-only task and the beginning of the track-and-count task. This gap is, as previously mentioned, the $5-\mathrm{sec}$ interval during which the subject receives the instructions for the counting task. Thus, there exists the possibility that the accommodative shift towards the visual far point is occurring not only during the counting task, but also when the subject is passively processing the instructions.

Also, separate Newman-Keuls tests were performed for the remaining target distances $(0.0 \mathrm{D}$ and $3.0 \mathrm{D})$ and tasks over the seven time blocks. Results from these tests indicated that each of the subjects' responses may be regarded as statistically flat. Whatever the interpretation of these results, it is, nevertheless, evident that the greatest accommodation shift away from the near target occurred, not while the subject was performing the backwards counting task, but during the 5 -sec time period when he was processing the secondary task instructions.

Again disregarding the open-loop accommodative shifts, there is a three-way interaction effect of Tasks by Target Distance by Time Blocks which is of central interest. Accommodation tends to shift during the counting task, and the shift intensifies as the counting task continues. This accommodative shift is entirely consistent with the unexpected results of the simulated night-landing experiment of Randle, Roscoe, and Pettit (Note 1). During their experiment, the pilots' accommodation was noted to push progressively towards the visual far point, according to the number of decisions and the amount of time devoted to the task.

Finally, with regard to the Target Distance Conditions by Time Blocks by Days interaction, it does seem that there is a certain amount of day-to-day fluctuation in the degree of accommodative shift observed. But, as this accommodative shift did not appear to involve any sequential trend over the days observed, it may well be that the effect does not involve any learning effects. Rather, there is an implication that any daily accommodative shift might well involve hitherto uncontrolled variables. Mood shifts have been suggested by both Miller (1978) and Roscoe and Benel (Note 3).

\section{EXPERIMENT 2: VISUAL IMAGERY TASK}

Experiment 1 questioned whether or not a concurrent, written counting task could bring about an accommodative shift. It seemed evident that the counting task brought about a progressive, cumu- 
lative shift in the direction of the visual far point, but there exists the possibility that the shift may have been brought about by the physical demands of the writing task or even by the instructional set. In addition, the first experiment involved a secondary task which was unrelated to the primary tracking task. And, finally, the open-loop dark-focus conditions yielded an unexpectedly large variance, which led to a decision to drop this condition from the next experiment.

A visual imagery task ("thinking near" and "thinking far") had previously been shown to induce small accommodative shifts in short-term (1 min or less) open-loop conditions (Malmstrom \& Randle, 1976). Also, because the visual imagery response had been observed to be slow and regular, it was considered an ideal response against which to compare the accommodative shift in the far direction described in Experiment 1. Furthermore, effects of visual imagery in the presence of a peripheral stimulus had yet to be determined. Therefore, it was decided to compare the effects of visual imagery in the presence of a foveal as well as a peripheral stimulus. The question addressed was whether the visual imagery response of thinking near and thinking far would override the stimuli in the visual field. Or, would the stimulus "visually capture" (Posner, Nissen, \& Klein, 1976) the accommodation response? If there were "visual capture," then the presence of a peripheral stimulus might be expected to have a smaller effect on the "visual capture." The results of an experiment by Weber and Malmstrom (1979) had suggested that the presence of an appropriate visual stimulus would allow eye movements to emerge during the course of a visual imagery task. However, the possibility of inhibition of an ocular motility response was not considered.

\section{Method}

Subjects. Four of the five subjects had participated in Experiment 1; a fifth subject was added after receiving comparable training on the optometer. This subjects's (J.N.) near and far points, as measured by the attached focus stimulator, are also shown in Table 1. All subjects were paid $\$ 3.50$ for two experimental $1 / 2-h$ sessions, and all were naive as to the visual imagery task.

Apparatus and Stimuli. All measurements were taken with the Cornsweet and Crane (1970) optometer, as described in Experiment 1. All subjects were presented with the following stimuli: (1) a peripheral target, a black-rimmed $O$ whose outer and inner dimensions were, respectively, 8.3 and $6.3 \mathrm{deg}$ of visual angle, and (2) a foveal target, a black " $x$ " whose visual angle subtended $2.9 \mathrm{deg}$. The target background brightness remained constant and at the same levels as in Experiment 1. The targets were held in constant focus using the Crane and Cornsweet (1970) focus stimulator.

Instructions and Procedure. The subjects were informed that this experiment involved their ability to imagine near and far objects. When the black $\times$ was presented, the subject was instructed to look at the center and to indicate when he felt it was in focus.
At that time, the subject was instructed to imagine the $x$ to be as near as possible, to imagine it as far away as possible, or merely to watch the target. When the black $O$ was presented, the subject was instructed to look at the edge, to indicate when he felt the $O$ was in focus, and then to shift his gaze to the center of the $O$. An equipment limitation of the Cornsweet and Crane optometer is that it cannot tolerate significant eye movements beyond about $15 \mathrm{~min}$ of arc. Hence, if the subject were to be looking anywhere other than the approximate center of the black $X$ or $O$, the accommodation response would be rendered unreadable. When the subject indicated that the $O$ was in focus, he was further instructed to imagine an object of his choice (such as a baseball) in the center of the $O$ to be coming towards him as close as possible, to be moving away from him as far as possible, or merely to be stationary in the center.

At no time during the experiment was the subject informed of the optical distance of the target; he was only told to imagine it to be either as near or as far away as possible, or else to just hold the target in focus. With the attached focus stimulator, all targets retained the same relative brightness, size, and contrast, so these factors could not be used as distance cues.

Each subject's responses were measured from the point he considered the O.O-D $\times$ target to be in focus; hence, all readings have been corrected for each subject's refractive error. Each reading was recorded continuously on a Brush recorder and measured to the nearest .1 D. A single trial lasted $35 \mathrm{sec}$, but each of the seven time blocks is actually an average of five instantaneous diopter readings taken from $2.5 \mathrm{sec}$ before each time block to $2.5 \mathrm{sec}$ after each time block.

Design. A 2 by 3 by 2 by 2 by 7 within-subjects design was employed, consisting of two peripheral-foveal target conditions (O, X target), three task instructions ("think near," "think far," "watch target"), two target distances $(0.0 \mathrm{D}, 3.0 \mathrm{D})$, two trials, and the seven time blocks $(0-30 \mathrm{sec})$. Each subject received all of the 12 conditions presented in random order; for the second trial, the original random order of presentation was reversed to counterbalance any learning effects.

\section{Results and Discussion}

Figures 2, 3, and 4 indicate the accommodation responses, respectively, for the imagery tasks of "think near," "think far," and "watch the target." The responses, under each of the tasks, are shown as functions of the target distances and time blocks. Peripheral targets are denoted by an $O$, and foveal targets are denoted by an $x$.

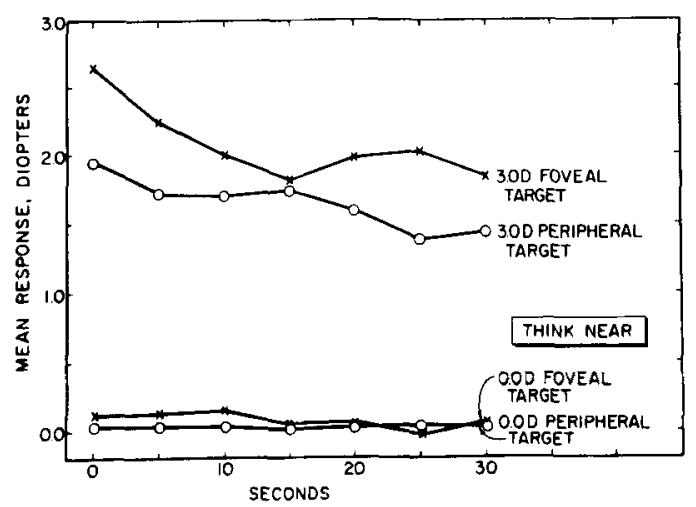

Figure 2. Experiment 2. Mean accommodation responses to instructions "think near" in the presence of both foveal and peripheral targets; $N=5$. 


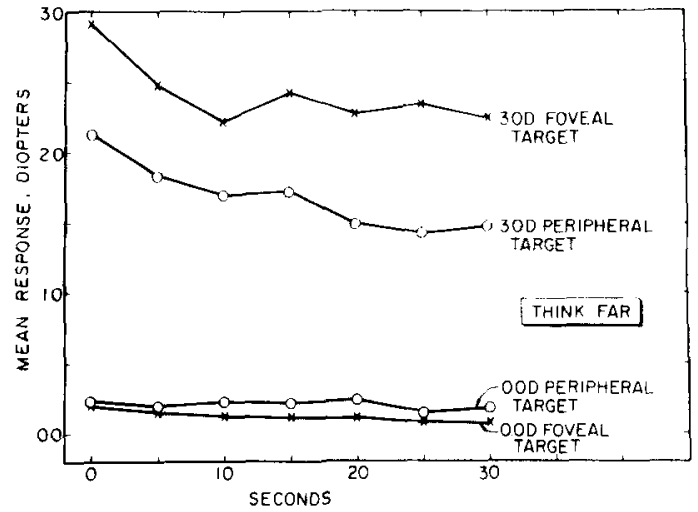

Figure 3. Experiment 2. Mean accommodation responses to instructions "think far" in the presence of both foveal and peripheral targets; $N=5$.

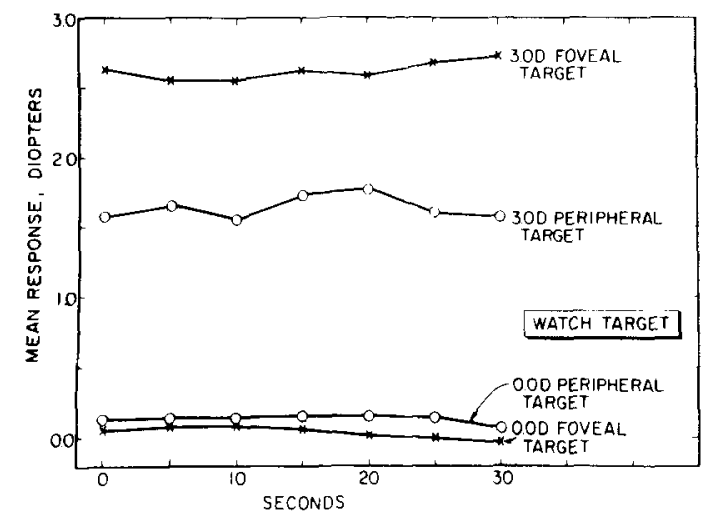

Figure 4. Experiment 2. Mean accommodation responses to instructions "watch target" in the presence of both foveal and peripheral targets; $N=5$.

As might be expected, as in Experiment 1, there is a large difference in the accommodation response which is dependent upon the optical distance at which the target is placed. Again, this is an expected and trivial effect, indicating that the eye tends to focus on whatever target happens to be in the field of view. Even so, there is an apparent tendency for the point of focus of the eye to wander away from the near (3.0 D) target, regardless of the task instructions.

There are also large differences between the accommodation responses to the foveal targets and those to the peripheral targets, and these peripheralfoveal response differences also tend to vary over time. Hence, it would appear that there is a greater tendency for dark focus (or instrument myopia) to occur in the presence of a peripheral stimulus (or aperture). Even so, these instrument myopia responses appear to be larger in the presence of a 3.0-D peripheral target than in the presence of a $0.0-\mathrm{D}$ target. These results are consistent with the previous findings of Hennessy (1975) and Hennessy et al. (1976).

As predicted by Posner, Nissen, and Klein (1976), there is no overall difference in the responses to in- structions to "think near, "think far," and "watch the target" in the presence of either a foveal or a peripheral stimulus. Therefore, it would appear that if perceptual and imaginal processes are placed in conflict, visual dominance results. Indeed, Malmstrom and Randle (1976) have previously shown that open-loop visual imagery responses are extremely small in comparison with stimulus field responses.

Of central interest is the indication that the concurrent imagery task has a time-dependent effect on accommodation. First, it appears that the imagery task effects are most noticeable when the target is near; a complimentary way of expressing this is that a stimulus placed at infinity focus has a better chance for "visual capture." Matthews, Angus, and Pearce (1978) have reported improving target detection by placing an accommodative aid at optical infinity in an otherwise empty field. Second, the effects of the imagery task on accommodation in the presence of a stimulus field appears to be in a very stereotyped direction. Over a $30-\mathrm{sec}$ period, regardless of whether the subject attempts to "think near" or "think far" in the presence of a 3.0-D stimulus field, he consistently tends to nudge his accommodation to the far direction. This unexpected and stereotyped response is quite different from that found in the previous study of Malmstrom and Randle (1976) in which subjects effected small, but appropriate, imagery-induced accommodation shifts in open-loop conditions.

Analysis of variance was performed using the accommodative response as the dependent variable. Main effects were found for the foveal-peripheral targets $[F(1,4)=10.324, p<.05]$, target distances $[\mathrm{F}(1,4)=23.478, \mathrm{p}<.01]$, and time blocks $[\mathrm{F}(6,24)$ $=3.927, \mathrm{p}<.01]$. The two-way interactions of Time Blocks by Distance $[\mathrm{F}(6,24)=3.326, \mathrm{p}<.02]$, Time Blocks by Task $[\mathrm{F}(12,48)=2.310, \mathrm{p}<.025]$, and Targets by Distance $[F(1,4)=31.369, p<.005]$ were also significant. In addition, there was a single significant three-way interaction of Time Blocks by Distance by Task $[\mathrm{F}(12,48)=2.927, \mathrm{p}<.01]$. No other main or interaction effects were observed.

In an effort to isolate the sources of variance, four additional analyses of variance were performed. The first two analyses compared separately the near and the far target responses. The analysis for the far target yielded a significant main effect for only the peripheral-foveal target $[\mathrm{F}(1,4)=8.053, \mathrm{p}<.05]$. Hence, it does appear that the effect of an aperture is solely responsible for the accommodation shift away from the far target towards the near direction (see Hennessy et al., 1976). Time and task effects are not significant relative to a near accommodation shift.

Analysis of variance for the near target yielded 
only the significant main effects: for the peripheral foveal targets $[F(1,4)=18.850, p<.025]$ and for time blocks $[\mathrm{F}(6,24)=3.802, \mathrm{p}<.01]$ and a single two-way interaction for Tasks by Time Blocks $[F(12,48)=2.877, p<.01]$. These results have several implications. First, the small-pupil or restingstate effect of the peripheral target on accommodation is stronger away from the near target than it is away from the far target. Second, the effect of the task is clearly away from the near target to the far direction. Furthermore, this task-dependent accommodative shift in the far direction is progressive over time.

In order to examine more closely the effects of the secondary task on the subjects' ability to maintain focus over individual time blocks, numerous Newman-Keuls tests were performed over the seven time blocks $(0-30 \mathrm{sec})$. When the foveal and peripheral target conditions are pooled, the means of the "watch-the-target" conditions appear to differ substantially from those of the "think-near" and "thinkfar" conditions. Newman-Keuls tests indicate that all the plots on the "watch-the-target" conditions (Figure 4) may be regarded as statistically flat. However, similar tests indicate that only Time Blocks 1 and 2 ( 0 and $5 \mathrm{sec}$ ) of the 3.0-D (near) target differ both from each other and from all successive time blocks $(p<.01)$. Such a finding implies that the greatest accommodative shift away from the near target occurs very early and rapidly during the introduction of the secondary task. This early occurrence of the accommodative shift would certainly be in line with the possibility that during Experiment 1 the greatest accommodative shift occurred while the subject was processing the secondary task instructions.

Finally, two additional analyses of variance were performed using only the near target accommodative responses, one for the foveal and one for the peripheral target. Curiously, neither of the analyses alone yielded an acceptable level of significance for an expected Task by Time Blocks interaction. (For both analyses, $.1>\mathrm{p}>$.05.) Therefore, the findings suggest that both the near peripheral and foveal targets are equally likely to allow an accommodative shift to the far direction. There was, however, a significant main effect for time blocks only in the presence of a near peripheral target, and this result again suggests that there is still a considerable amount of accommodative wander away from a near peripheral target. For this analysis, $F(6,24)=5.553, \mathrm{p}<.001$. Nevertheless, this indication should be tempered by the fact that the Newman-Keuls test failed to find any significant differences between data points when the near foveal and peripheral target means were pooled under the "watch-the-target" conditions. Long-term open-loop accommodative wander cer- tainly will require greater scrutiny in subsequent experiments.

\section{GENERAL DISCUSSION}

In both Experiment 1 and Experiment 2 there is a remarkably similar three-way interaction effect of Task by Target Distance by Time Blocks. This accommodation shift is both progressive and in the direction of the visual far point. Furthermore, this effect is strongest in the presence of a near, foveal stimulus; under open-loop or peripheral target conditions, the effect is somewhat questionable, or at least more subject to unaccounted-for variables. The question necessarily arises as to whether the subjects' accommodation shift was towards the visual far point or towards the dark focus. This question cannot, however, be answered simply. First, the accommodation shift under open-loop conditions was observed to be far too unstable to allow for any degree of a confident conclusion. There could be several sources of this dark-focus instability - the open loop (vs. the illumination) method of determination and the long- (vs. the short-) term wander effects, also described by Roscoe and Benel (Note 3).

In regard to the stimulus field accommodative shift to the far direction, however, the results are less equivocal. In Experiment 2, the accommodative shift observed away from the far target towards the visual near point was attributable only to the effects of the peripheral stimulus, not to the nature of the secondary task. Furthermore, two subjects (D.S. and R.L.), whose dark foci were, respectively, 2.83 and $1.93 \mathrm{D}$ (see Table 1), exhibited no tendency to shift their accommodation toward these values during concurrent mental tasks.

Why this accommodation shift towards the far point should occur most readily in the presence of a near visual stimulus is by no means clear. There are at least two contemporary models that could account for this shift. An attentional or capacity model, described by Kahneman (1973), in which inattention to the visual stimulus because of effort expended on the secondary task, would seem to be a likely candidate. However, this model does not account for the absence of an accommodative shift away from the far target to the dark focus. A second model that might describe the accommodation shift is the autonomic arousal model of Hess and Polt (1964). Certainly, the direction of the accommodation shift is consistent with the expected pupillary response during the performance of a secondary task. It must also be stated that we are not prepared to speculate whether this observed accommodative shift is a phenomenon in its own right or was induced by an involuntary pupillary convergence response; neither 
the convergence nor the pupillometric responses were measured in this study. In the future, any taskinduced interactions of accommodation, convergence, and pupillometric response ought to be well worth examining.

There is a progressive, time-dependent effect of the concurrent mental task on accommodation which has several implications. First, most measurements of accommodation have previously assumed static conditions, both of the target and of the eye. In the real world, this is rarely so, for, as our results indicate, changes in accommodation are not merely a function of the target stimulus movement. Second, the observation that accommodation pushes towards the visual far point as a function of the duration of concurrent mental activity would suggest a convenient explanation for eye fatigue. The exact human adaptive value of such a cumulative accommodative shift is not readily evident. However, from an evolutionary standpoint, it could be argued that intensive periods of mental activity, coupled with the necessity to maintain extended near focus (such as in reading or mathematical problem solving) are only very recent human endeavors. If it could be shown that, say, extended periods of reading and concentration were to involve a constant battle of an ongoing accommodation shift in the far direction and a recurring attempt to eliminate the retinual blur from the shift, such an opponent process would go a considerable distance towards explaining subjective reports of eye fatigue (asthenopia). In the past, reports of reading eye fatigue have been ascribed to eye-muscle fatigue, or even "subjective reports of mild discomfort." (See Tinker, 1958, and Smith, 1979, for a review of this subject.) Historically, the explanations have been both unsatisfactory and insufficient.

A further word of caution is in order. The authors suggest only that an objective measure of accommodation (i.e., lens curvature) is affected by a concurrent mental task. Absolutely no claim is made that target perception is altered, although this possibility certainly needs to be examined.

In summary, there appear to be at least three separate ways of inducing the accommodative shift to the far direction in the presence of a near visual stimulus. These ways are: a concurrent mental counting task, a concurrent mental imagery task, and, perhaps, the process of responding to the instructional set. In all conditions, the shift appeared to be between .25 and $.75 \mathrm{D}$ away from the near target, although the shift increased as the secondary task continued. The results of these experiments are consistent with both the capacity model of Kahneman (1973) and the autonomic arousal model of Hess and Polt (1964). There does not seem to be evidence in these experiments that will distinguish clearly be- tween these alternative models. It should also be noted that neither of these experiments discounts the possibility of an intermediate resting position of accommodation model, but only that a concurrent mental task may be a questionable method of inducing the dark focus.

\section{REFERENCE NOTES}

1. Randle, R., Roscoe, S., \& Petitt, J. Effects of accomodation and magnification on aim-point estimation in a simulated landing task. NASA Tech. Paper 1635, October 1980.

2. Malmstrom, F., Randle, R., \& Weber, R. Perceptual tunnel or perceptual sphere? Accommodation and stress. Paper presented at the meeting of the Rocky Mountain Psychological Association, Salt Lake City, Utah, May 7-10, 1975.

3. Roscoe, S., \& Benel, R. Is the eye smart or the brain forgiving? (AFOSR Tech. Rep. 78-1). Urbana-Champaign: Department of Psychology, University of Illinois, April 1978.

\section{REFERENCES}

Ahern, S., \& Beatty, J. Pupillary responses during information processing vary with scholastic aptitude test scores. Science, $1979,205,1289-1292$.

CogAN, D. Accommodation and the autonomic nervous system. Archives of Ophthalmology, 1937, 18, 739-766.

Cornsweet, T., \& Crane, H. Servo-controlled infrared optometer. Journal of the Optical Society of America, 1970, 60, 548-554.

Crane, H., \& Cornsweet, T. Ocular-focus stimulator. Journal of the Optical Society of America, 1970, 60, 577.

Davson, H. Physiology of the eye (3rd ed.). New York: Academic Press, 1976.

Hennessy, R. Instrument myopia. Journal of the Optical Society of America, 1975, 65, 1114-1120.

Hennessy, R., Iida, T., Shina, K., \& Leibowitz, H. The effect of pupil size on accommodation. Vision Research, 1976, 16, 587-589.

Hess, E. The tell-tale eye. New York: Van Nostrand Reinhold, 1975.

Hess, E., \& Polt, J. Pupil size as related to interest value of visual stimuli. Science, 1960, 132, 349-350.

Hess, E, \& Polt, J. Pupil size in relation to mental activity during simple problem-solving. Science, 1964, 143, 1190-1192.

Kahneman, D. Attention and effort. Englewood Cliffs, N.J: Prentice-Hall, 1973.

Kahneman, D., \& Beatty, J. Pupil diameter and load on memory. Science, 1966, 145, 1583-1585.

Leibowitz, H., \& Owens, D. Anomalous myopias and the intermediate dark focus of accommodation. Science, 1975, 189, 646-648.

Malmstrom, F., \& Randle, R. Effects of visual imagery on the accommodation response. Perception \& Psychophysics, $1976,19,450-453$.

Matthews, M., Angus, R., \& Pearce, D. Effectiveness of accommodative aids in reducing empty field myopia in visual search. Human Factors, 1978, 20, 733-740.

Miller, R. Mood changes and the dark focus of accommodation. Perception \& Psychophysics, 1978, 24, 437-443.

Morgan, M., Olmsted, R., \&. Watrous, W. Sympathetic action in accommodation for far vision. American Journal of Physiol$o g y, 1940,128,588-591$.

OGi.e, K. Optics. Springfield, Ill: Thomas, 1961.

Oimsten, J., \& Morcian, M. Refraction of the rabbit's eye in 
the unexcited and excited state. American Journal of Physiology, $1939,127,602-604$.

Owens, D. The Mandelbaum effect: Evidence for an accommodative bias toward intermediate viewing distances. Journal of the Optical Society of America, 1979, 83, 157-171.

Posner, M., Nissen, M., \& Klein, R. Visual dominance: An information-processing account of its origins and significance. Psychological Review, 1976, 83, 157-171.

Randle, $R$. The non-visual in visual accommodation. In Proceedings, IEEE International Conference on Cybernetics and Society, San Francisco, September 23-25, 1975.

Smith, W. A review of literature relating to visual fatigue. In Proceedings of the Human Factors Society, October 29-November 1, 1979, pp. 362-366.
SсновеR, H. Über die Akkommodationsruhelage [On the resting position of accommodation]. Optik, 1954, 11, 282-290.

Tinker, M. Recent studies of eye movements in reading. Psychological Bulletin, 1958, 55, 215-231.

Weber, R., \& Malmstrom, F. Measuring the size of mental images. Journal of Experimental Psychology: Human Perception and Performance, 1979, 5, 1-12.

Westheimer, G. Accommodation measurements in empty fields. Journal of the Optical Society of America, 1957, 47, 714-718.

(Received for publication April 5, 1979; revision accepted July 24,1980 .) 